\title{
Comparative Gene Expression of Intestinal Metabolizing Enzymes
}

\author{
Ho-Chul Shin ${ }^{\mathrm{a}, *}$, Hye-Ryoung Kim ${ }^{\mathrm{a}}$, Hee-Jung Cho ${ }^{\mathrm{a}}$, Hee $\mathrm{Yi}^{\mathrm{a}}$, Soo-Min Cho ${ }^{\mathrm{a}}$, Dong-Goo Lee ${ }^{\mathrm{a}}$, \\ A. M. Abd El-Aty ${ }^{a}$, Jin-Suk Kim ${ }^{a}$, Duxin Sun ${ }^{b}$ and Gordon L . Amidon ${ }^{b}$ \\ a Department of Veterinary Pharmacology and Toxicology, Konkuk University, Seoul 143-701, Republic of Korea \\ ${ }^{\mathrm{b}}$ Department of Pharmaceutical Sciences, College of Pharmacy, University of Michigan, Ann Arbor, MI 48109, USA
}

\begin{abstract}
The purpose of this study was to compare the expression profiles of drugmetabolizing enzymes in the intestine of mouse, rat and human. Total RNA was isolated from the duodenum and the mRNA expression was measured using Affymetrix GeneChip oligonucleotide arrays. Detected genes from the intestine of mouse, rat and human were ca. $60 \%$ of 22690 sequences, $40 \%$ of 8739 and $47 \%$ of 12559 , respectively. Total genes of metabolizing enzymes subjected in this study were 95, 33 and 68 genes in mouse, rat and human, respectively. Of phase I enzymes, the mouse exhibited abundant gene expressions for Cyp3a25, Cyp4v3, Cyp2d26, followed by Cyp2b20, Cyp2c65 and Cyp4f14, whereas, the rat showed higher expression profiles of Cyp3a9, Cyp2b19, Cyp4f1, Cyp17a1, Cyp2d18, Cyp27a1 and Cyp4f6. However, the highly expressed P450 enzymes were CYP3A4, CYP3A5, CYP4F3, CYP2C18, CYP2C9, CYP2D6, CYP3A7, CYP11B1 and CYP2B6 in the human. For phase II enzymes, glucuronosyltransferase Ugtla6, glutathione S-transferases Gstp1, Gstm3 and Gsta2, sulfotransferase Sult1b1 and acyltransferase Dgat1 were highly expressed in the mouse. The rat revealed predominant expression of glucuronosyltransferases Ugt1a1 and Ugt1a7, sulfotransferase Sult1b1, acetyltransferase Dlat and acyltransferase Dgat1. On the other hand, in human, glucuronosyltransferases UGT2B15 and UGT2B17, glutathione S-transferases MGST3, GSTP1, GSTA2 and GSTM4, sulfotransferases ST1A3 and SULT1A2, acetyltransferases SAT1 and CRAT, and acyltransferase AGPAT2 were dominantly detected. Therefore, current data indicated substantial interspecies differences in the pattern of intestinal gene expression both for P450 enzymes and phase II drug-metabolizing enzymes. This genomic database is expected to improve our understanding of interspecies variations in estimating intestinal prehepatic clearance of oral drugs. Copyright (C) 2009 John Wiley \& Sons, Ltd.
\end{abstract}

Key words: gene expression; metabolizing enzymes; intestine; mouse/rat/human

\section{Introduction}

Recent advances in genomic technology have facilitated the rapid and simultaneous determination of global profiles for thousands of genes in tissue samples directly associated with the

*Correspondence to: Department of Veterinary Pharmacology and Toxicology, Konkuk University, Seoul 143-701, Republic of Korea. E-mail: hshin@konkuk.ac.kr pharmacokinetics [1-5]. The completion of the DNA sequencing of human, mouse and rat genomes and knowledge of cross-species gene homologies enables studies of differential gene expression in animal models [6]. Recent targeting of specific tissue genes, such as intestinal transporters or enzymes, has shown a potential to greatly enhance our understanding of pharmacokinetics including bioavailability and metabolic clearance for oral drug molecules [7-12]. It is now 
accepted that the drug absorption process from the intestine is highly associated with the functional gene expression of intestinal transporters and metabolizing enzymes $[7,13]$.

Many drugs are chemically altered in the body by various reactions of metabolizing enzymes. The key human enzyme subfamilies include CYP1A, CYP2A, CYP2B, CYP2C, CYP2E and CYP3A. It has been also generally understood that the major metabolizing enzymes are CYP3A4, CYP2D6, CYP2C8/9 and CYP1A2 in human. Each enzyme subfamily possesses specific selectivity toward substrate drugs. CYP3A4 shows mostly wide spectra for the metabolism of numerous drugs [14,15]. CYP2D6 metabolizes amoxapine, chloropromazine, and codeine, and CYP2C8/9 metabolizes phenytoin, ketamine, paclitaxel, rifampin and various sulfonamides. Although the enzymes are predominantly found in the liver, the intestinal enzymes also deserve a special mechanism for modulating drug bioavailability (the main contributor of prehepatic clearance of drugs) [11]. However, little is known about the interspecies differences in the distribution pattern of individual isoforms and functions at the level of mRNA expression for the intestinal metabolizing enzymes. Therefore, the genomic comparison of intestinal enzymes among some species is very valuable for interspecies prediction of drug bioavailability and animal scaling for first-pass metabolism capacity. This study provides a comparative transcriptional database for intestinal metabolizing enzymes between model rodent animals and human.

\section{Materials and Methods}

\section{Materials}

TRIzol reagent and superscript Choice System for cDNA synthesis kit were purchased from Invitrogen (Carlsbad, CA). The BioArray high-yield RNA transcript labeling kit was purchased from Enzo Biochem (New York, NY). The RNeasy kit was obtained from Qiagen (Valencia, CA). GeneChips including MG 430A 2.0 for mouse, RG U34A for rat and HG U95A for human were purchased from Affymetrix (Santa Clara, CA). The GeneChip hybridization and scanning for
RG U34A and HG U95A assays were performed at the Genomic Information Support Facility at Michigan State University (East Lansing, MI), and those for MG 430A 2.0 were carried out at the Seoulin Molecular Biology Technique Center (Seoul, Korea). Human biopsy samples were taken from the duodenum with an endoscope as described earlier $[7,16]$.

\section{Animals}

Male ICR mice (32-40g) were obtained from Orient BioCo. (Seoul, Korea) and housed in a controlled animal room of Konkuk University, Seoul, Korea. The mice were fed solid pellets with water ad libitum. Male SD rats (250-300 g) were purchased from Charles River laboratories (Wilmington, MA) and kept at the animal facility of the University of Michigan. The rat diets were purchased from TestDiet Inc (Richmond, IN).

\section{RNA isolation}

The human duodenal samples were prepared from healthy male and female volunteers (21-45 years of age, $n=10$ ) as described earlier [16]. All mucosal tissues of the rat $(n=8)$ and mouse duodenum $(n=6)$ were immediately scraped with a glass slide, transferred to a new frozen vial and dipped into liquid nitrogen. A total of $100 \mathrm{mg}$ of tissue was added to $1 \mathrm{ml}$ TRIzol reagent, homogenized with a tissue razor at maximum speed for $20 \mathrm{~s} \times 3$ times or with a mortar on ice. The homogenate was transferred to a new tube and then $200 \mu \mathrm{l}$ chloroform was added to the TRIzol mixture. After being centrifuged at $12500 \mathrm{rpm}$ for $15 \mathrm{~min}$ at $4^{\circ} \mathrm{C}$, the aqueous phase was transferred to a new tube, and the RNA was precipitated with $500 \mu \mathrm{l}$ of isopropanol, and washed with $80 \%$ ethanol. The RNA pellet was resuspended in $30-50 \mu \mathrm{l}$ of DNase/RNase-free water, and the concentration was measured at $260 \mathrm{~nm}$. The absorbance ratio at wavelength $260 / 280 \mathrm{~nm}$ should be at least greater than 1.6. The RNA was further purified with an RNease Mini kit (Qiagen, Valencia, CA) according to the manufacturer's manual. The concentration of the purified RNA was measured at $260 \mathrm{~nm}$. The absorbance ratio at $260 / 280 \mathrm{~nm}$ of the purified RNA should be 1.7-1.8. The purified RNA was checked in agarose/formaldehyde gel 
for quality before further cRNA labeling. Five $\mu \mathrm{g}$ of purified RNA was mixed with RNA loading buffer and heated at $75^{\circ} \mathrm{C}$ for $15 \mathrm{~min}$. After cooling down on ice for $5 \mathrm{~min}$, the RNA was loaded on $1 \%$ agarose/formaldehyde gel in $1 \mathrm{X}$ MOPS buffer. The gel was run at $80-100 \mathrm{~V}$ for $50 \mathrm{~min}$ and two sharp $18 \mathrm{~S}$ and $28 \mathrm{~S}$ bands should be visible under UV.

\section{Microarray assay}

Probe synthesis from total RNA samples, hybridization, detection and scanning were performed according to the standard protocols from Affymetrix, Inc. (Santa Clara, CA). In the rat and human, single stranded cDNA was transcribed from 5-8 $\mu$ g total RNA using T7-(dT) ${ }_{24}$ oligomer primer, the primer was annealed at $70^{\circ} \mathrm{C}$ for $10 \mathrm{~min}$, and then Superscript II reverse transcriptase was used for reverse transcription at $42^{\circ} \mathrm{C}$ for $1 \mathrm{~h}$. The double stranded cDNA was synthesized from first-strand cDNA using DNA ligase, DNA polymerase I and T4 DNA polymerase at $16^{\circ} \mathrm{C}$ for $2 \mathrm{~h}$ using SuperScript Choice System for cDNA synthesis kit (Gibco/BRL, Grand Island, NY, USA). The reaction was stopped by adding $10 \mu \mathrm{l}$ of $0.5 \mathrm{~m}$ EDTA. The double strand cDNA was then cleaned by phenol/chloroform extraction with phase-locking gel and ethanol precipitation in the presence of $1 \mu \mathrm{g}$ of glycogen. Biotin-labeled cRNA was synthesized from the double strand cDNA using T7 RNA polymerasecatalysed in vitro transcription in the presence of biotin-labeled NTP (BioArray high yield RNA transcription labeling kit, Enzo Biochem, New York, NY) at $37^{\circ} \mathrm{C}$ for $5 \mathrm{~h}$, and the labeled cRNA was purified using RNease mini kit (Qiagen). The concentration of labeled cRNA was measured at $260 \mathrm{~nm}$. A total of $20 \mu \mathrm{g}$ of labeled cRNA was fragmented at $95^{\circ} \mathrm{C}$. Biotinlabeled cRNA was heated at $99^{\circ} \mathrm{C}$ for $5 \mathrm{~min}$ in hybridization cocktail including hybridization control (Bio B, C, D and Cre) and hybridized with GeneChip ${ }^{\circledR}$ (Affymetrix, Santa Clara, CA) at $42^{\circ} \mathrm{C}$ for $16 \mathrm{~h}$. The GeneChip ${ }^{\circledR}$ was then washed with non-stringent wash buffer at $50^{\circ} \mathrm{C}$ and stained with streptavidin phycoerythrin (SAPE) solution. After washing at $25^{\circ} \mathrm{C}$, the GeneChip ${ }^{\mathbb{R}}$ was scanned with a laser scanner (Affymetrix). In mouse, cDNA was synthesised using the
One-Cycle cDNA Synthesis Kit (Affymetrix, Santa Clara, CA). Single stranded cDNA was synthesised using Superscript II reverse transcriptase and T7-oligo(dT) primers at $42^{\circ} \mathrm{C}$ for $1 \mathrm{~h}$. Double stranded cDNA was obtained by using DNA ligase, DNA polymerase I and RNase $\mathrm{H}$ at $16^{\circ} \mathrm{C}$ for $2 \mathrm{~h}$, followed by T4 DNA polymerase at $16^{\circ} \mathrm{C}$ for $5 \mathrm{~min}$. After clean up with a Sample Cleanup Module (Affymetrix, Santa Clara, CA), ds-cDNA was used for in vitro transcription (IVT). cDNA was transcribed using the GeneChip IVT Labeling Kit (Affymetrix, Santa Clara, CA), in the presence of biotinlabelled CTP and UTP. After clean up with a Sample Cleanup Molule (Affymetrix, Santa Clara, CA), the biotin-labelled IVT-RNA was fragmented. Fragmented cRNA was hybridized to the mouse gene chip MG $430 \mathrm{~A} 2.0$ at $45^{\circ} \mathrm{C}$ for $16 \mathrm{~h}$ according to the manufacturer's instructions. After hybridization, the arrays were washed in a GeneChip Fluidics Station 450 with a nonstringent wash buffer at $25^{\circ} \mathrm{C}$ followed by a stringent wash buffer at $50^{\circ} \mathrm{C}$. After washing, the arrays were stained with a streptavidin-phycoerythrin complex. Afterwards, they were processed according to the procedure described above for rat and human array. The duodenal mRNA expression profile obtained for SLC15A1 from microarray data analyses was validated using semiquantitative RT-PCR. The RT-PCR assay was performed as described previously [16]. SLC15A1 mRNA expression in the individual biopsies determined by RT-PCR exhibited a pattern similar to that observed with the microarray data $\left(r^{2}=0.89\right)$.

\section{Data analysis}

Official symbols and gene names were used in accordance with the symbol and name lists approved by HUGO (Human Genome Organization) Gene Nomenclature Committee (http:// www.genenames.org). Data analysis was performed using different software packages including Microarray Suite (Affymetrix, Santa Clara, CA) and GeneSpring 6.2 (Silicon Genetics, Redwood City, CA). Numeric data were extracted from DAT images and normalized using the Microarray Suite. Gene function analysis was performed using the gene ontology-mining tool of NetAffx, which is

Biopharm. Drug Dispos. 30: 411-421 (2009) DOI: $10.1002 / \mathrm{bdd}$ 
based on the Gene Ontology database (http:// www.geneontology.org). GeneSpring also uses data found publicly in genomics databases to build gene ontologies based on annotation information. For the present GeneChip probe array study, the data for each gene represent data from 11-20 probe pairs each approximately $25 \mathrm{bp}$ in length. For each probe pair, one probe is a perfect match while the other has a single mismatch at nucleotide 13. The mismatch probe works as an internal control to evaluate the cross hybridizations between closely related target sequences. The overall target-specific intensity was obtained by the difference between the intensity of perfect match and the mismatch probes. GeneChip Operating Software (GCOS, Affymetrix, CA) was used to determine the absolute analysis metrics (Detection, Detection $p$-value) using the scanned probe array data and compared between the different treatment group signals to generate the Change, Change $p$-value, and Signal log ratio (fold change). For normalization, data from each expression array were scaled, so that the overall fluorescence intensity across each chip was equivalent (average target intensity set at 500). The 'Selected Probe Sets' method was applied using the normalization control probe sets. Equivalency for arrays being compared was assessed based on the scaling/normalization factors calculated by the 'Selected Probe Sets' (GCOS, Affymetrix, CA). The one-sided Wilcoxon's signed rank test was employed to generate the detection $p$ value. If the overall intensity of perfect match was much larger than that of mismatch, the detection $p$ value would be small. The probe set would be regarded as present if the $p$-value was less than 0.04 and if the $p$-value was higher than 0.06 , the probe set would be regarded as absent. The detection threshold was set as the 'present' call output from GCOS $(p<0.05)$. Two sets of algorithms were generated and they were used to generate change significance and change quantity metrics for every probe set. The change algorithm generated a Change $p$-value and an associated fold-change value. The second algorithm gave a quantitative estimate of the change in gene expression in the form of Signal Log Ratio. The level of gene expression was regarded as increased if its Change $p$-value was less than 0.0025 and the gene expression would be considered to be decreased if its Change $p$-value was greater than 0.9975 . Only relative changes equal or greater than 2-fold level of expression were considered. For a given gene transcript in any chip-to-chip comparison, GCOS generates a 'change call' parameter ('increase' or 'decrease') based on a consideration of signal specificity as well as intensity.

\section{Results}

Expression profiles of 22690 gene sequences in mouse intestine, 8739 in rat and 12559 in human were analysed. Detected genes were ca. $60 \%, 40 \%$ and $47 \%$ from mouse, rat and human intestine, respectively. This paper focused on the expression of metabolizing enzymes as critical factors modulating prehepatic clearance of many oral substrates. As shown in Table 1, the total numbers of metabolizing enzymes detected were 95, 33 and 68 genes in mouse, rat and human intestine, respectively. Relatively higher intensities of gene expression were observed in glucuronosyltransferases and acyltransferases.

Figure 1 shows the expression of P450s. Several types of Сyp3a including Сyp3a25, Cyp3a9 and Cyp3a4 were dominantly expressed in mouse, rat and human. Expression intensities

Table 1. The number of genes expressed in mouse, rat and human intestine

\begin{tabular}{|c|c|c|c|c|c|c|}
\hline \multirow[t]{2}{*}{ Gene group } & \multicolumn{2}{|c|}{ Mouse } & \multicolumn{2}{|c|}{ Rat } & \multicolumn{2}{|c|}{ Human } \\
\hline & $\begin{array}{l}\text { Detected } \\
\text { genes }\end{array}$ & $\begin{array}{l}\text { Average } \\
\text { intensity }\end{array}$ & $\begin{array}{l}\text { Detected } \\
\text { genes }\end{array}$ & $\begin{array}{l}\text { Average } \\
\text { intensity }\end{array}$ & $\begin{array}{l}\text { Detected } \\
\text { genes }\end{array}$ & $\begin{array}{l}\text { Average } \\
\text { intensity }\end{array}$ \\
\hline Cytochrome P450s & 30 & 71 & 16 & 862 & 24 & 275 \\
\hline Glucuronosyltransferases & 5 & 786 & 7 & 2391 & 6 & 2144 \\
\hline Glutathione S-transferases & 19 & 253 & 1 & 150 & 9 & 1145 \\
\hline Sulfotransferases & 15 & 84 & 3 & 1167 & 15 & 422 \\
\hline Acetyltransferases & 15 & 71 & 4 & 583 & 9 & 882 \\
\hline Acyltransferases & 11 & 232 & 2 & 12950 & 5 & 1474 \\
\hline
\end{tabular}

Copyright (C) 2009 John Wiley \& Sons, Ltd. 


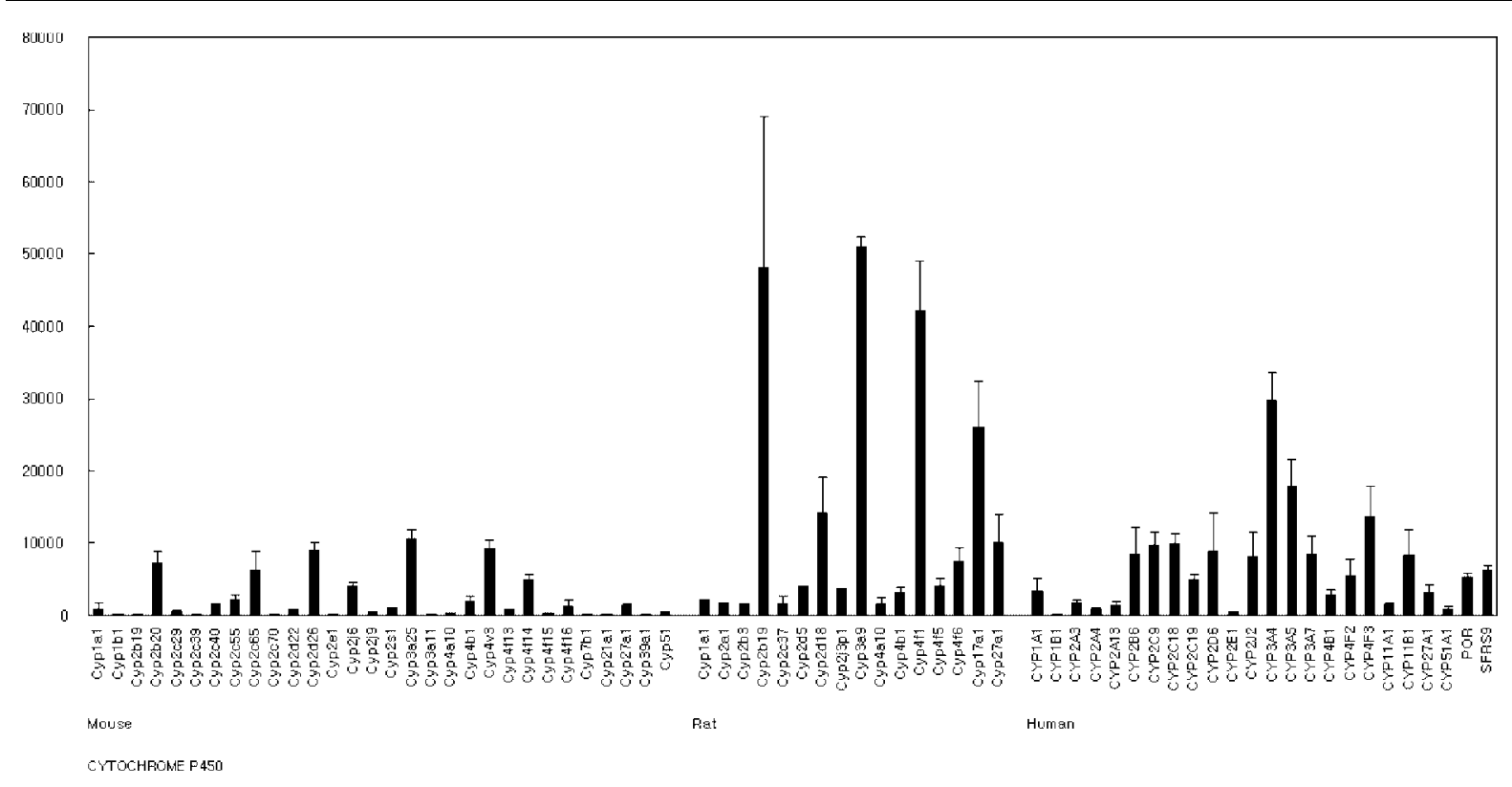

Figure 1. Comparative gene expression for various cytochrome P450s in the intestine of mice, rats and humans $(n>6)$. Expression levels were measured by the intensity of hybridization signal using GeneChip array. (CYP1A1, cytochrome P450, family 1 , subfamily a, polypeptide 1 )

of cytochrome P450s were in order of Cyp3a25 $>$ Cyp4v3 > Cyp2d26 > Cyp2b20 > Cyp2c65 > Cyp4f14 in mouse, Cyp3a9 > Cyp2b19 > Cyp4f1 > Cyp17a1 $>$ Cyp2d18 $>$ Cyp27a1 in rat, and CYP3A4 $>$ CYP3A5 > CYP4F3 > CYP2C18 > CYP2C9 > CYP2D6 > CYP3A7 > CYP11B1 > CYP2B6 in human. The expressions of other P450s were minor or negligible. Of UDP-glucuronsyltransferases (UGT), higher expression levels were observed in Ugt1a6 (mouse), Ugt1a1 and Ugt1a7 (rat) and UGT2B15 and UGT2B17 of human compared with the other UGTs (Figure 2). As shown in Figure 3, expression intensity of glutathione S-transferases was in the order Gstp1 > Gstm3 > Gsta2 > Mgst3 > Mgst1 > Gsto1 > Gstm1 > Mgst2 in mouse, and MGST3 > GSTP1 $>$ GSTA2 > GSTM4 > MGST2 > GSTM1 in human. Figure 4 shows that sulfotransferases had a similar gene expression pattern between mouse and rat. Sult1b1 was distinctly expressed both in mouse and rat, but not detected in human. Human revealed a dramatically higher intensity of ST1A3 and SULT1A2 expression. Of the acetyltransferases (Figure 5), Acat1 and Sat1 were highly expressed in mouse, Dlat in rat, and SAT1 and CART in human. Figure 6 shows the acyltransferase expression levels in these species. Rat showed a very

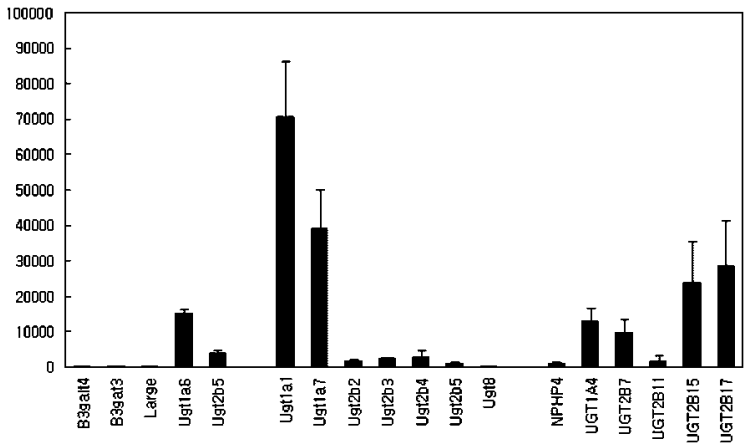

Figure 2. Comparative gene expression for various glucuronosyltransferases in the intestine of mice, rats and humans $(n>6)$. Expression levels were measured by the intensity of hybridization signal using GeneChip array. (UGT1A1, UDP-glucuronosyltransferase 1a1; UGT1A4, bilirubin UDP-glucuronosyltransferase isozyme 2; UGT1A6, UDP-glucuronosyltransferase 1 family, polypeptide A6; UGT1A7, UDP-glucuronosyltransferase 1a7; UGT2B2, androsterone UDP-glucuronosyltransferase; UGT2B3, liver 17 beta-hydroxysteroid UDP-glucuronosyltransferase; UGT2B4, UDP-glucuronosyltransferase 2b4; UGT2B5, UDPglucuronosyltransferase 2 family, member 5; UGT2B7, 3,4catechol estrogen UDP-glucuronosyltransferase; UGT2B11, UDP-glucuronosyltransferase 2B; UGT2B15, UDP glucuronosyltransferase; UGT2B17, C19steroid specific UDP-glucuronosyltransferase; UGT8, UDP-glucuronosyltransferase 8; B3GALT4, UDP-Gal:betaGalNAc beta 1,3-galactosyltransferase, polypeptide 4; B3GAT3, beta-1,3-glucuronyltransferase 3 (glucuronosyltransferase I); LARGE, like-glycosyltransferase; NPHP4, AKIAA0673 protein) 


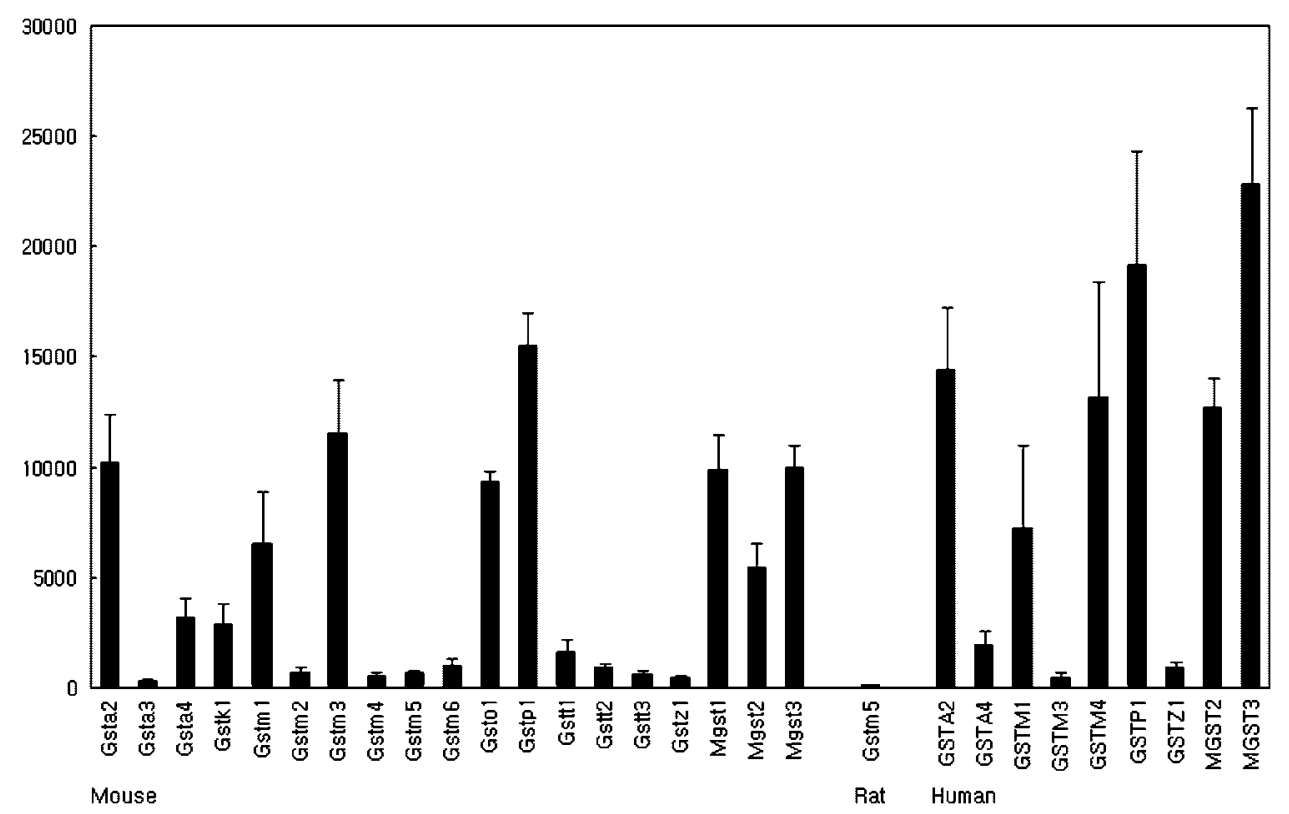

Figure 3. Comparative gene expression for various glutathione S-transferases in the intestine of mice, rats and humans $(n>6)$. Expression levels were measured by the intensity of hybridization signal using GeneChip array. (GSTA2-4, glutathione Stransferase, alpha 2-4; GSTK1, glutathione S-transferase, kappa 1; GSTM1-6, glutathione S-transferase, mu 1-6; GSTO1, glutathione S-transferase, omega 1; GSTP1, glutathione S-transferase, pi 2; GSTT1-3, glutathione S-transferase, theta 1-3; GSTZ1, glutathione transferase, zeta 1 (maleylacetoacetate isomerase); MGST1-3, microsomal glutathione S-transferase 1-3)

high expression intensity of Dgat1, and mouse expressed Dgat1 and Acaa2 at high levels. In human, AGPAT2 and AGPAT1 were found at higher levels.

\section{Discussion}

It was widely recognized that there were marked inter- or intraspecies differences in distribution and functions of each metabolizing enzyme class including cytochrome P450s and transferases. However, since these studies focused on the hepatic metabolism [17], there has been very poor information regarding other tissues such as intestine. Therefore, it is necessary to develop P450 and transferase-based differential screening systems that may able to predict the risk and pharmacological responses of xenobiotics between animals and humans. Recently, these screening systems are being accomplished by use of genomic techniques. Molecular probes are being used to screen the levels of gene expression of drug-related enzymes in an effort to determine whether expression of certain P450 forms or transferases is associated with increased drug discovery and risk assessment [1,2].

Cytochrome P450s, a class of phase I metabolizing enzymes, are dominantly expressed in liver and intestine to regulate metabolism and oral bioavailability, and these enzymes have shown a very wide range of substrate specificities $[18,19]$. For this reason, examining the differences in the expression levels of these enzymes among some species may provide insight into the differences in $\mathrm{PK}$ and drug bioavailability properties. Up to now, there are more than 71 characterized cytochrome P450 genes by HUGO. These enzymes are responsible for the metabolism (at least partial metabolism) of approximately $75 \%$ of all drugs, with the CYP3A subfamily being responsible for nearly half of this activity $[20,21]$. Because of their wide substrate spectrums and potential for numerous drug-drug interactions in both preclinical and clinical studies, these subfamilies CYP1A, CYP2A, CYP2B, CYP2D, CYP2E and CYP3A are considered as key enzymes [21-23]. In our study, mice showed primary expression of Cyp3a25, Cyp4v3 and Cyp2d26, and rats revealed higher 


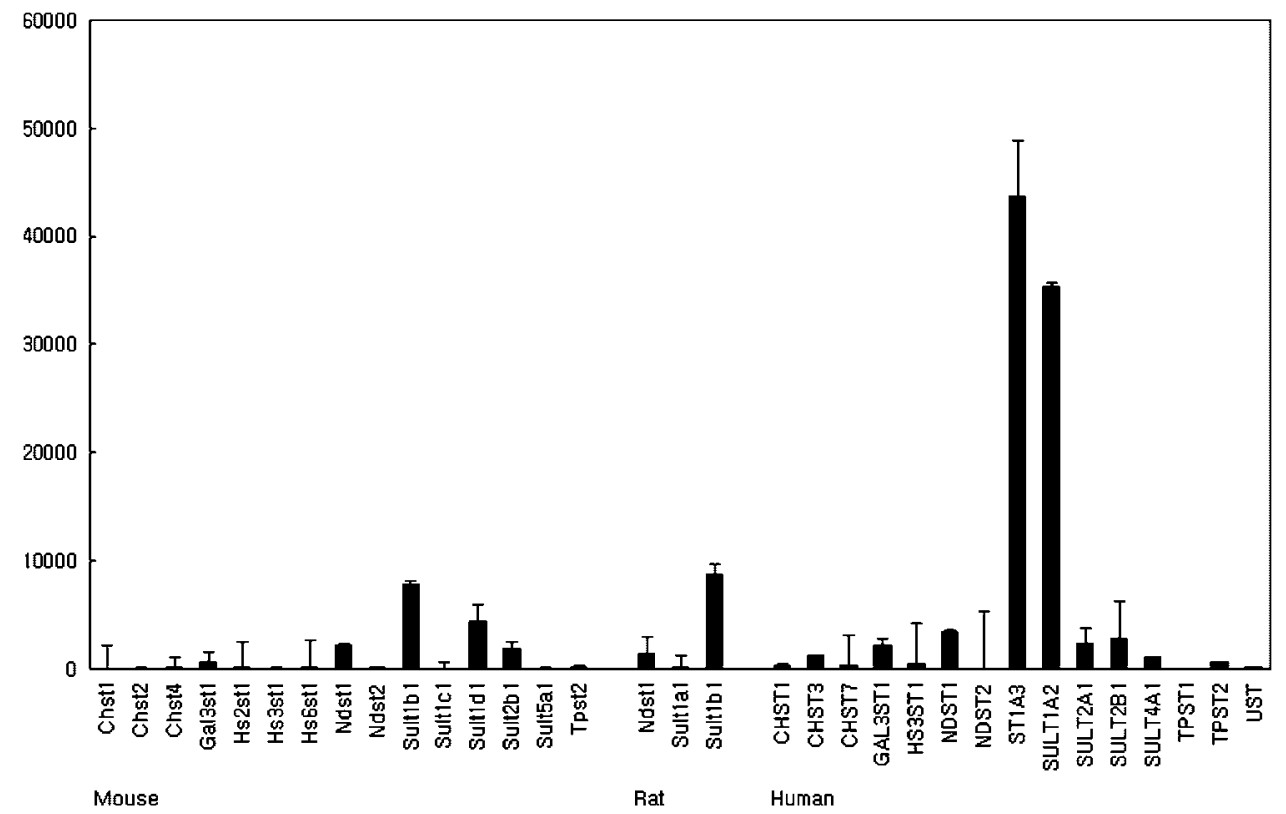

Figure 4. Comparative gene expression for various sulfotransferases in the intestine of mice, rats and humans $(n>6)$. Expression levels were measured by the intensity of hybridization signal using GeneChip array. (CHST1-4, carbohydrate (keratan sulfate Gal-6) sulfotransferase 1-4; CHST7, chondroitin 6-sulfotransferase like protein; GAL3ST1, galactosylceramide sulfotransferase; HS2ST1, heparan sulfate 2-O-sulfotransferase 1; HS3ST1, heparan sulfate (glucosamine) 3-O-sulfotransferase 1; HS6ST1, heparan sulfate 6-O-sulfotransferase 1; NDST1-2, N-deacetylase/N-sulfotransferase (heparan glucosaminyl) 1-2; SULT1A1, minoxidil sulfotransferase; SULT1A2, thermostable phenol sulfotransferase (STP2); SULT1B1, sulfotransferase family 1B, member 1; SULT1C1, sulfotransferase family, cytosolic, 1C, member 1; SULT1D1, sulfotransferase family 1D, member 1; SULT2A1, dehydroepiandrosterone sulfotransferase (STD) gene; SULT2B1, sulfotransferase family, cytosolic, 2B, member 1; SULT4A1, sulfotransferase (Sulfokinase) like gene; SULT5A1, sulfotransferase family 5A, member 1; TPST2, protein-tyrosine sulfotransferase 2; ST1A3, aryl sulfotransferase; TPST1, tyrosylprotein sulfotransferase-1; TPST2, tyrosylprotein sulfotransferase; UST, dermatan/chondroitin sulfate 2-sulfotransferase)

transcription of Cyp3a9, Cyp2b19, Cyp4f1 and Cyp17a1. On the other hand, humans have dominantly expressed CYP3A4, CYP3A5, CYP4F3 and CYP2C subfamilies. Expression intensities of the other minor P450 genes were also considerably different among these species. Significant expression of Cyp3a25 in mouse intestine has been also reported by earlier investigators [24,25], though an identification study of intestinal P450s has not been well described in mouse intestine compared with rat and human. It is well known that the CYP3A4 is a predominant P450 enzyme in human intestine, and Cyp3a9 in rat intestine [26,27]. Wacher et al. [28] reported that CYP3A4 in human intestine plays a significant role in the first-pass metabolism of $50-70 \%$ of marketed drugs. Zierold et al. [29] and Xue et al. [30] reported that the human CYP3A4 is thought to be the homolog of the rat Cyp3a9, though the two enzymes are not identical because of four different amino acids in the substrate recognition site. Human CYP3A4 reveals higher homology of DNA sequence with rat Cyp3a9 (81\%) than that of mouse Cyp3a25 (76\%). These data might indicate that there are extensive interspecies variations in the preference of substrate structure recognition, though few members of CYP3A subfamily, including Сyp3a25, Сyp3a9 and CYP3A4, are expressed as the principal gene in these species. Therefore, this result may partially explain 'why the oral first-pass metabolism of many substrates differs in these species'.

Probably the most common conjugation reaction is the synthesis of glucuronic acid derivatives (glucuronides). Aliphatic alcohols, phenols, carboxylic acids, mercaptans, primary and secondary aliphatic amines, and carbamates are 


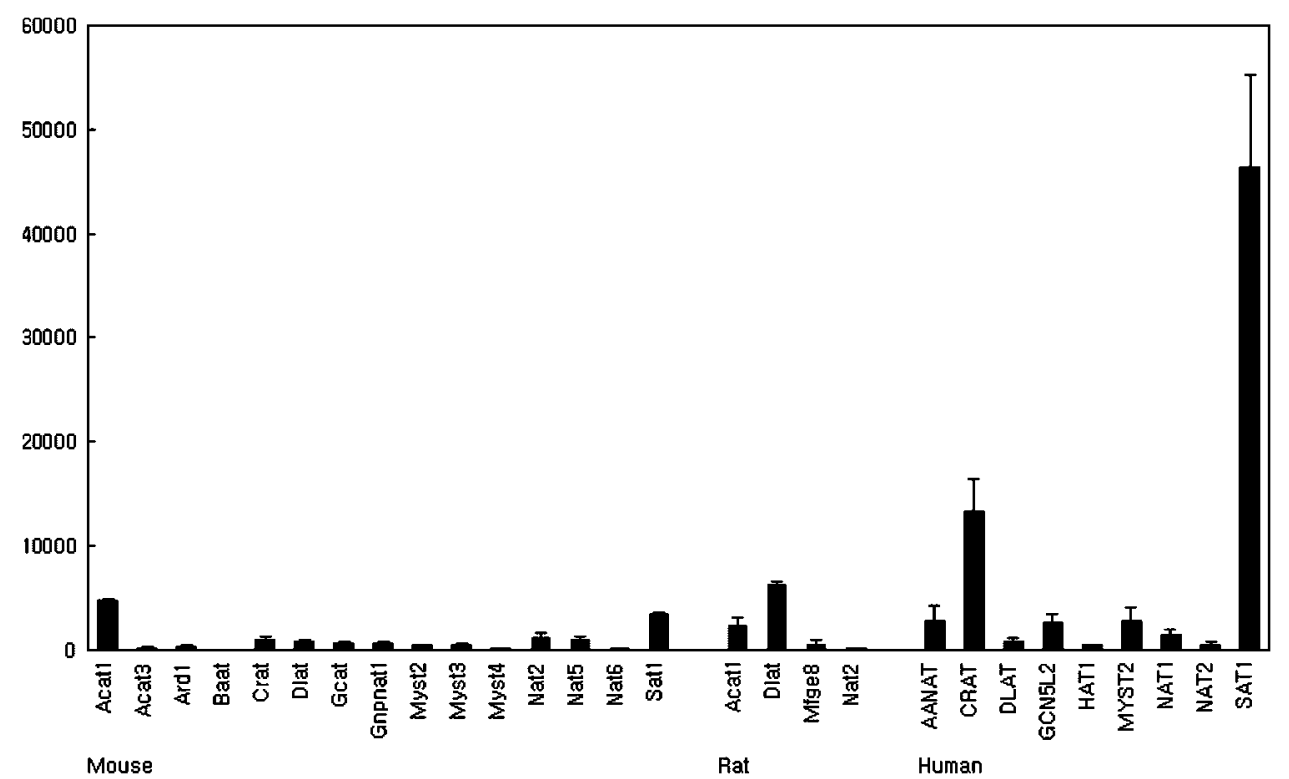

Figure 5. Comparative gene expression for various acetyltransferases in the intestine of mice, rats and humans ( $n>6)$. Expression levels were measured by the intensity of hybridization signal using GeneChip array. (ACAT1, acetyl-coenzyme A acetyltransferase 1; ACAT3, acetyl-coenzyme A acetyltransferase 3; ARD1, N-acetyltransferase ARD1 homolog (S. cerevisiae); BAAT, bile acid-coenzyme A: amino acid N-acyltransferase; CRAT, carnitine acetyltransferase; DLAT, dihydrolipoamide Sacetyltransferase; GCAT, glycine C-acetyltransferase (2-amino-3-ketobutyrate-coenzyme A ligase); GNPNAT1, glucosaminephosphate $N$-acetyltransferase 1; MYST2-4, MYST histone acetyltransferase 2-4; NAT1, arylamine N-acetyltransferase; NAT2, $\mathrm{N}$-acetyltransferase 2 (arylamine $\mathrm{N}$-acetyltransferase); NAT5, $\mathrm{N}$-acetyltransferase 5 (ARD1 homolog, S. cerevisiae); NAT6, Nacetyltransferase 6; SAT1, spermidine/spermine N1-acetyl transferase 1; MFGE8, O-acetyltransferase; AANAT, serotonin Nacetyltransferase gene; GCN5L2, histone acetyltransferase; HAT1, histone acetyltransferase 1)

converted to their beta-glucuronide derivatives by UDP-glucuronosyltransferase (UGT). In our study, higher expression levels were observed in Ugt1a6 of mouse, Ugt1a1 and Ugt1a7 of rat, and UGT2B15 and UGT2B17 of human when compared with other glucuronosyltransferases. Glutathione S-transferases are ubiquitous multifunctional proteins involved in the detoxification of endogenous and xenobiotic compounds using either glutathione conjugation or glutathione peroxidase activity [31,32]. The major roles of GSTs are the metabolism of electrophilic carcinogens and alkene and arene oxides [33]. The data show interspecies variability for the expression pattern of glutathione S-transferases. Expression intensity was high in Sgtp1, Gstm3, Gsta2, Mgst3, Mgst1 and Gsto1 in mouse, and MGST3, GSTP1, GSTA2, GSTM4 and MGST2 in human. However, in rats, the enzyme Gstm5 was merely observed at the lower level. Sulfotransferase (SULT) catalyses the transfer of a sulfate group to the hydroxyl group of drug compounds [34]. Sult1b1 was distinctly expressed in both mouse and rat, but not detected in humans. On the other hand, human revealed a dramatically higher intensity of ST1A3 and SULT1A2 expression. It can be summarized that sulfotransferases have a similar gene expression pattern between mouse and rat, but not human. Acetylation of drug compounds is catalysed in human and animals by various types of acetyltransferases such as MYST histone acetyltransferases (MYSTs), N-acetyltransferases (NATs) and spermidine/spermine N1-acetyltransferases (SATs). The data have shown a variety of gene expression profiles for acetyltransferases depending on the species. Acat1 and Sat1 were highly expressed in mouse, Dlat in rat, and SAT1 and CART in human in which SAT1 expression level was 3-4 fold higher than CART. Several types of xenobiotic carboxylic acids including aromatic, heteroaromatic, arylacetic, cinnamic and aryloxyacetic are conjugated with a variety of endogenous amino acids including glycine, 


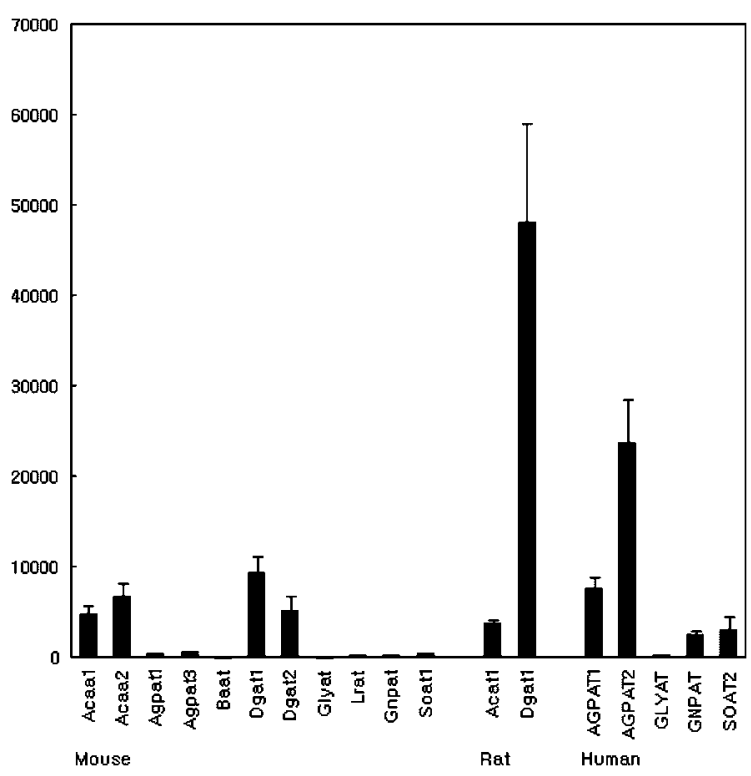

Figure 6. Comparative gene expression for various acyltransferases in the intestine of mice, rats and humans $(n>6)$. Expression levels were measured by the intensity of hybridization signal using GeneChip array. (ACAA1-2, acetyl-coenzyme A acyltransferase 1-2; AGPAT1, 1-acylglycerol-3-phosphate Oacyltransferase 1 (lysophosphatidic acid acyltransferase, alpha); AGPAT2, lysophosphatidic acid acyltransferase-beta; AGPAT3, 1-acylglycerol-3-phosphate O-acyltransferase 3; BAAT, bile acid-coenzyme A: amino acid $N$-acyltransferase; DGAT1, diacylglycerol O-acyltransferase 1; DGAT2, diacylglycerol Oacyltransferase 2; GLYAT, glycine- $N$-acyltransferase; LRAT, lecithin-retinol acyltransferase (phosphatidylcholine-retinol-Oacyltransferase); GNPAT, glyceronephosphate O-acyltransferase; SOAT1, sterol O-acyltransferase 1; ACAT1, acetyl-CoA acyltransferase)

glutamine and taurine, prior to excretion in mammals [35]. It was observed that the acyltransferase expression levels are extensively different in mouse, rat and human. Rat showed a very high expression intensity of Dgat, and mouse expressed Dgat1 and Acaa2 at high levels. However, in human, AGPAT2 and AGPAT1 were found as the primary enzyme genes compared with other acyltransferases.

The potential role of intestinal enzymes on drug first-pass metabolism has become increasingly recognized. Although animal models do not provide direct information that can be extrapolated to humans, drug metabolism studies will all benefit from the selection of animal models species that closely approximates human metabolism. Since it is very difficult technically

Copyright (C) 2009 John Wiley \& Sons, Ltd. to assay the expression of whole enzymes at proteomic levels, it is readily accepted that the gene expression studies using microarray technology is an essential tool for predicting functional expression of intestinal proteins. It is still arguable that the transcriptional profile may not be consistent with functional protein levels. Nevertheless, several recent investigations have revealed positive correlation between mRNA expression and regulation of functional proteins [7,36-40]. Berggren et al. [39] reported that mRNA level of CYP3A4 was significantly correlated with its protein levels in small intestine. Overall, the data reveal that rodent animals and humans have a variety of different transcriptional profiles for metabolizing enzymes in the small intestine. To our knowledge, there has been no report directly describing interspecies difference in intestinal expression of metabolism enzymes at genomic levels. Therefore, this transcriptional data can lead to more effective use of animal models in oral drug development and application to human.

\section{Acknowledgements}

This work was partially supported by the Korea Research Foundation Grant (KRF-2007-331E00251).

\section{References}

1. Norinder U. In silico modelling of ADMET - a minireview of work from 2000 to 2004. SAR QSAR Environ Res 2005; 16: 1-11.

2. van de Waterbeemd H, Gifford E. ADMET in silico modelling: towards prediction paradise? Nat Rev Drug Discov 2003; 2: 192-204.

3. Clark DE, Grootenhuis PD. Progress in computational methods for the prediction of ADMET properties. Curr Opin Drug Discov Devel 2002; 5: 382-390.

4. Fiegler H, Carter NP. Genomic array technology. Methods Cell Biol 2004; 75: 769-785.

5. Anderle P, Huang Y, Sadee W. Intestinal membrane transport of drugs and nutrients: genomics of membrane transporters using expression microarrays. Eur J Pharm Sci 2004; 21: 17-24.

6. Fang H, Tong W, Perkins R, et al. Bioinformatics approaches for cross-species liver cancer analysis

Biopharm. Drug Dispos. 30: 411-421 (2009)

DOI: $10.1002 / \mathrm{bdd}$ 
based on microarray gene expression profiling. BMC Bioinformatics 2005; 6(Suppl 2): S6.

7. Landowski CP, Sun D, Foster DR, et al. Gene expression in the human intestine and correlation with oral valacyclovir pharmacokinetic parameters. J Pharmacol Exp Ther 2003; 306: 778-786.

8. Takara K, Ohnishi N, Horibe S, Yokoyama T. Expression profiles of drug-metabolizing enzyme CYP3A and drug efflux transporter multidrug resistance 1 subfamily mRNAS in small intestine. Drug Metab Dispos 2003; 31: 1235-1239.

9. Rushmore TH, Kong AN. Pharmacogenomics, regulation and signaling pathways of phase I and II drug metabolizing enzymes. Curr Drug Metab 2002; 3: 481-490.

10. Mizuno N, Niwa T, Yotsumoto $Y$, Sugiyama $\mathrm{Y}$. Impact of drug transporter studies on drug discovery and development. Pharmacol Rev 2003; 55: 425-461.

11. Beaumont K. The importance of gut wall metabolism in determining drug bioavailability. In Drug Bioavailability/Estimation of Solubility, Permeability and Absorption. Volume 18, Methods and Principles in Medicinal Chemistry, Waterbeemd HV-d, Lennernas H, Artursson P (eds). Weinheim: Wiley-VCH, 2003; 311-328.

12. Tsuji A. Transporter-mediated drug interactions. Drug Metab Pharmacokinet 2002; 17: 253-274.

13. Cao X, Gibbs ST, Fang L, et al. Why is it challenging to predict intestinal drug absorption and oral bioavailability in human using rat model. Pharm Res 2006; 23: 1675-1686.

14. $\mathrm{Bu} \mathrm{HZ}$. A literature review of enzyme kinetic parameters for CYP3A4-mediated metabolic reactions of 113 drugs in human liver microsomes: structure-kinetics relationship assessment. Curr Drug Metab 2006; 7: 231-249.

15. Berecz R, Dorado P, De La Rubia A, et al. The role of cytochrome P450 enzymes in the metabolism of risperidone and its clinical relevance for drug interactions. Curr Drug Targets 2004; 5: 573-579.

16. Sun D, Lennernas H, Welage LS, et al. Comparison of human duodenum and Caco-2 gene expression profiles for 12000 gene sequences tags and correlation with permeability of 26 drugs. Pharm Res 2002; 19: 1400-1416.

17. Spatzenegger M, Jaeger W. Clinical importance of hepatic cytochrome P450 in drug metabolism. Drug Metab Rev 1995; 27: 397-417.

18. Watkins PB. Drug metabolism by cytochromes P450 in the liver and small bowel. Gastroenterol Clin North Am 1992; 21: 511-526.

19. Fujita K. Cytochrome P450 and anticancer drugs. Curr Drug Metab 2006; 7: 23-37.

20. Kaminsky LS, Fasco MJ. Small intestinal cytochromes P450. Crit Rev Toxicol 1991; 21: 407-422.

Copyright (C) 2009 John Wiley \& Sons, Ltd.
21. Anzenbacher P, Anzenbacherova E. Cytochromes P450 and metabolism of xenobiotics. Cell Mol Life Sci 2001; 58: 737-747.

22. Michalets EL. Update: clinically significant cytochrome P-450 drug interactions. Pharmacotherapy 1998; 18: 84-112.

23. Sitkiewicz D. Mechanisms of drug metabolism implications for drug interaction. Pol Merkuriusz Lek 2000; 9: 595-597.

24. Martignoni M, Groothuis G, de Kanter R. Comparison of mouse and rat cytochrome P450mediated metabolism in liver and intestine. Drug Metab Dispos 2006; 34: 1047-1054.

25. Zhang QY, Dunbar D, Kaminsky LS. Characterization of mouse small intestinal cytochrome P450 expression. Drug Metab Dispos 2003; 31: 1346-1351.

26. Kato M. Intestinal first-pass metabolism of CYP3A4 substrates. Drug Metab Pharmacokinet 2008; 23: 87-94.

27. Shayeganpour A, El-Kadi AO, Brocks DR. Determination of the enzyme(s) involved in the metabolism of amiodarone in liver and intestine of rat: the contribution of cytochrome P450 3A isoforms. Drug Metab Dispos 2006; 34: 43-50.

28. Wacher VJ, Silverman JA, Zhang Y, Benet LZ. Role of P-glycoprotein and cytochrome P450 $3 \mathrm{~A}$ in limiting oral absorption of peptides and peptidomimetics. J Pharm Sci 1998; 87: 1322-1330.

29. Zierold C, Mings JA, Deluca HF. 19nor-1,25Dihydroxyvitamin D2 specifically induces CYP3A9 in rat intestine more strongly than 1,25dihydroxyvitamin D3 in vivo and in vitro. Mol Pharmacol 2006; 69: 1740-1747.

30. Xue L, Zgoda VG, Arison B, Almira Correia M. Structure-function relationships of rat liver CYP3A9 to its human liver orthologs: sitedirected active site mutagenesis to a progesterone dihydroxylase. Arch Biochem Biophys 2003; 409: 113-126.

31. Salinas AE, Wong MG. Glutathione S-transferases - a review. Curr Med Chem 1999; 6: 279-309.

32. Whalen R, Boyer TD. Human glutathione Stransferases. Semin Liver Dis 1998; 18: 345-358.

33. Chasseaud LF. The role of glutathione and glutathione S-transferases in the metabolism of chemical carcinogens and other electrophilic agents. Adv Cancer Res 1979; 29: 175-274.

34. Rath VL, Verdugo D, Hemmerich S. Sulfotransferase structural biology and inhibitor discovery. Drug Discov Today 2004; 9: 1003-1011.

35. Caldwell J. Conjugation reactions in foreigncompound metabolism: definition, consequences, and species variations. Drug Metab Rev 1982; 13: 745-777.

36. Cox B, Kislinger T, Emili A. Integrating gene and protein expression data: pattern analysis and profile mining. Methods 2005; 35: 303-314.

Biopharm. Drug Dispos. 30: 411-421 (2009)

DOI: $10.1002 / \mathrm{bdd}$ 
37. Greenbaum D, Jansen R, Gerstein M. Analysis of mRNA expression and protein abundance data: an approach for the comparison of the enrichment of features in the cellular population of proteins and transcripts. Bioinformatics 2002; 18: 585-596.

38. Terada T, Inui K. Gene expression and regulation of drug transporters in the intestine and kidney. Biochem Pharmacol 2007; 73: 440-449.
39. Berggren S, Gall C, Wollnitz N, et al. Gene and protein expression of P-glycoprotein, MRP1, MRP2, and CYP3A4 in the small and large human intestine. Mol Pharm 2007; 4: 252-257.

40. Kern W, Kohlmann A, Wuchter C, et al. Correlation of protein expression and gene expression in acute leukemia. Cytometry B Clin Cytom 2003; 55: 29-36. 